\title{
Post-Publication Review
}

Salim, S. A., Sedera, D., Sawang, S., Alarifi, A. H. E., \& Atapattu, M. (2015). Moving from evaluation to trial: How do SMEs start adopting cloud ERP? Australasian J ournal of Information Systems, 19, S219-S254. doi: http:// dx.doi.org/ 10.3127/ ajis.v19i0.1030

\section{Review}

Cloud-based enterprise resource planning (ERP) is an approach to business process management with low subscription overhead that uses a network of remote servers hosted on the Internet to provide businesses with a system for automating business functions (e.g. accounting, human resources, inventory, order management). By improving the accessibility of internal data (employees can upload and access company data in real time via the cloud from any device with an Internet connection), cloud ERP provides work flexibility and mobility and enables greater collaboration among employees from both the sameand different departments than traditional on-premises ERP solutions.

Recognizing that ERP adoption in business settings is complex and thus goes beyond a single activity, Salim et al. (2015) sought to understand the adoption process of cloud ERP and the critical adoption factors in the evaluation and trial stages of the adoption process. The studypremised on the theory of planned behavior (TPB), Ettlie's multi-stage adoption model, and a sample of 162 owners of small and medium-sized enterprises (SMEs)-is novel and makes important contributions by identifying potential differences in significance and impact of factors that influence the intention of SMEs to adopt cloud ERP across the two adoptive stages.

Salim et al.'s (2015) article offers several positive takeaways:

1. The article is exemplary in its articulation of the research problem in terms of the limitations of existing studies (i.e. simple technology adoption, technology adoption within a single broad stage) and the critical need for greater clarity on the factors that influence complex technology adoption (e.g. cloud ERP; i.e. to improve decision making, to understand changes in critical adoption factors, to understand the requirements for adequate vendor involvement, and to understand the rationales for switching vendors or dropping out, all of which pertain to the complex technology adoption process; Dubey and Wagle 2007; Law and Ngai 2007; Muscatello et al. 2003; Willcocks and Sykes 2000; Winters et al. 2008).

2. The article makes a good case for examining cloud ERP in the context of SMEs-that is, the accessibility to (and benefits derived from) a complete range of IT applications that were once restricted to larger organizations - and is supported by recent statistics showing significant adoption intentions.

3. The article provides a good explanation of its contributions to theory and practice by addressing the identified research problems in the context of SMEs' adoption of cloud ERP. That is, by combining two complementary theories in the form of TPB and Ettlie's multi-stage adoption model, the study demonstrates the multi-stage process of cloud ERP adoption and contributes to the understanding of the different levels of significance of each factor in each of the adoption stages, including the reasons certain factors are more or less prominent in a particular stage. Note that though only two of the five adoption stages (i.e. evaluation and trial stages) were studied, the article provides ample support from previous studies to justify such a narrow focus of investigation (i.e. most critical stages in the process of adoption, reasons for adoption dropouts related to limited information from these two stages, apparent differences before and after experiencing cloud ERP, and completion of stages leading to actual decision on cloud ERP usage; Arthur 1989; Au and Kauffman 2003; Howard and Sheth 1969). 
4. The article is based on solid theoretical foundations. Up-to-date (i.e. 1967- 2015) studies related to technology adoption were covered in detail, a thorough explanation of the adoption stages was provided (i.e. awareness, interest, evaluation, trial, and commitment), determinants of decision making were adopted from a widely used behavioral model (i.e. TPB; attitude, subjective norms, perceived behavioral control, and intention), and adequaterationales from previous studies were used to support five research propositions under study (i.e. attitudes, subjective normal, perceived behavioral control, intention, and construct fluctuations between evaluation and trial stages in the adoption process).

5. The article is rigorous and practice oriented in the methods used and analysis conducted. That is, constructs were developed using closely related studies (e.g. Ettlie 1980; Fichman and Kemerer 1997; Harrison et al. 1997), appropriate guidelines were used for identifying formative variables (i.e. do not need to covary, must not be interchangeable, must cause the core construct and not be caused by it, and must allow variability in antecedents and consequences under different nomological nets; Cenfetelli and Bassellier 2009; J arvis et al. 2003; Petter et al. 2007), practical relevance for using formative constructs were provided (i.e. specific and actionable attributes of formative constructs, as weight of constructs can be used to draw practical implications on the importance of specific details; Furneaux and Wade 2011; Mathieson et al. 2001), a pretest (with a sample of 30 respondents) was carried out to ensure adequate reliability and validity of items measuring constructs before full-scale survey administration, sampling criteria were transparent (e.g. 162 Malaysian SMEs were selected according to the National SME Development Council's definition of SMEs, key informants were owners of SMEs whose opinions were deemed as representing the voice of the entire firm, questionnaires were administered at a conference targeted to SMEs, participation was voluntary and no incentives were provided), and the partial least square technique of structural equation modeling was justified (e.g. purpose of study involves predictive ability of constructs rather than theoretical validation, small sample size; Hair et al. 2011) and rigorously conducted (i.e. assessment of internal consistency, discriminant and convergent validity, and multicollinearity and use of Bonferroni-corrected p-value to guard against global type I error; Diamantopoulos and Winklhofer 2001; Fornell and Larcker 1981; Gable et al. 2008; Nunnally and Bernstein 1991; Shaffer 1995).

6. The article offers a meaningful discussion of the implications of the findings to theory and practice. The study extends the assumption of technology adoption from a singlestage process to a multi-stage process (theory), extends the scope of TPB (i.e. cloud ERP as a new research context, evidence of negative perceived behavioral control-intention relationship, modeling of TPB in a multi-stage process, and support for treating TPB components such as subjective norms as formative constructs), and provides the cloud ERP and SME ecosystem with a nuanced understanding of potential users' perceptions of and behaviors toward the adoption of cloud ERP (practice).

Nonetheless, in addition to the five limitations acknowledged (i.e. limited to evaluation and trial stages in the adoption process, limited explanation on intention- behavior relationship, limited in generalizability to other types of complex technology adoption, limited to SMEs whose important decisions are made by one individual, and limited to SMEs in Malaysia), Salim et al.'s (2015) work contains two notable shortcomings that pave the way for further research in the area:

1. Although the study reports some differences between critical adoption factors in evaluation and trial stages of the adoption process for cloud ERP among SMEs, these differences were only analyzed descriptively. Moreover, observation of path coefficients $(\beta)$ imply that identified differences (e.g. between the attitude-intention and subjective norms- intention paths) might not be significant (vs. significance of paths when taken individually) as a result of marginal improvements between predictors (e.g. in the evaluation stage, the difference in $\beta$ between the attitude-intention and subjective 
norms- intention paths is only 0.023 [i.e. 0.385 - 0.362]). Thus, further research can improve the rigor of analysis for multi-stage adoption processes by testing for significant differences in impact (or significance of improvements) between critical adoption factors within and across stages.

2. Although the critical adoption factors are well grounded in TPB and items measuring the constructs are adapted from existing studies in the area, an optimistic outlook suggests that more can be done to identify and examine factors that are more contextualized in order to induce greater adoption of cloud ERP among SMEs. For example, a qualitative study can be conducted to explore from SME owners' (and employees') perspectives the facilitators and barriers to cloud ERP adoption, which can subsequently be tested quantitatively (and potentially as an elaborated part of perceived behavioral control).

In short, Salim et al.'s (2015) article is a well-written piece whose positive takeaways can be used as a guide for writing a good research paper. In addition, their article goes beyond addressing important gaps in current understanding by offering new pathways for theoretical and practical extensions in the area. Thus, further research can improve the adoption of and reduce the attrition rates of cloud ERP in business settings using the avenues discussed both in Salim et al.'s (2015) article and in this post-publication review.

\section{Weng Marc Lim}

Swinburne University of Technology (Sarawak Campus) wlim@swinburne.edu.my

\section{References}

Arthur, W. B. (1989). Competing technologies, increasing returns, and lock-in by historical events. The Economic J ournal, 99 (394), 116-131.

Au, Y. A., \& Kauffman, R. J . (2003). What do you know? Rational expectations in information technology adoption and investment. J ournal of Management Information Systems, 20 (2), 49-76.

Cenfetelli, R. T., \& Bassellier, G. (2009). Interpretation of formative measurement in information systems research. MIS Quarterly, 33 (4), 689-707.

Diamantopoulos, A., \& Winklhofer, H. M. (2011). Index construction with formative indicators: An alternative to scale development. J ournal of Marketing Research, 19 (2), 269-277.

Dubey, A., \& Wagle, D. (2007). Delivering software as a service. The McKinsey Quarterly. Available

at http://www.lcis.com.tw/paper_store/paper_store/ Delivering_software_as_a_service20154515250718.pdf

Ettlie, J. E. (1980). Adequacy of stage models for decisions on adoption of innovation. Psychological Reports, 46 (3), 991-995.

Fichman, R. G., \& Kemerer, C. F. (1997). The assimilation of software process innovations: An organizational learning perspective. Management Science, 43 (10), 1345-1363.

Fornell, C., \& Larcker, D. F. (1981). Evaluating structural equation models with unobservable variables and measurement error. J ournal of Marketing Research, 19 (1), 39-50.

Furneaux, B., \& Wade, M. (2011). An exploration of organizational level information systems discontinuance intentions. MIS Quarterly, 35 (3), 573-598.

Gable, G. G., Sedera, D., \& Chan, T. (2008). Re-conceptualizing information system success: The IS-impact measurement model. Journal of the Association for Information Systems, 9 (7), 377-408. 
Hair, J . F., Ringle, C. M., \& Sarstedt, M. (2011). PLS-SEM: Indeed a silver bullet. J ournal of Marketing Theory and Practice, 19 (2), 139-152.

Harrison, D. A., Mykytyn J r., P. P., \&Riemenschneider, C. K. (1997). Executive decisions about adoption of information technology in small business: Theory and empirical tests. Information Systems Research, 8 (2), 171-195.

Howard, J . A., \& Sheth, J . N. (1969). The theory of buyer behavior. NewYork: Wiley.

Jarvis, C. B., MacKenzie, S. B., \& Podsakoff, P. M. (2003). A critical review of construct indicators and measurement model misspecification in marketing and consumer research. J ournal of Consumer Research, 30 (2), 199-218.

Law, C. C., \& Ngai, E. W. (2007). ERP systems adoption: An exploratory study of the organizational factors and impacts of ERP success. Information and Management, 44 (4), 418-432.

Mathieson, K., Peacock, E., \&Chin, W. W. (2001). Extending the technology acceptancemodel: The influence of perceived user resources. ACM SigMIS Database, 32 (3), 86-112.

Muscatello, J . R., Small, M. H., \& Chen, J. J . (2003). Implementing enterprise resource planning (ERP) systems in small and midsize manufacturing firms. International J ournal of Operations and Production Management, 23 (8), 850-871.

Nunnally, J . C., \&Bernstein, I. H. (1991). Psychometric theory. New York: McGraw-Hill.

Petter, S., Straub, D., \& Rai, A. (2007). Specifying formative constructs in information systems research. MIS Quarterly, 31 (4), 623-656.

Salim, S. A., Sedera, D., Sawang, S., Alarifi, A. H. E., \& Atapattu, M. (2015). Moving from evaluation to trial: How do SMEs start adopting cloud ERP? Australasian J ournal of Information Systems, 19, S219-S254. doi: http:/ / dx.doi.org/ 10.3127/ ajis.v19i0.1030

Shaffer, J . P. (1995). Multiple hypothesis testing. Annual Review of Psychology, 46 (1), 561584.

Willcocks, L. P., \& Sykes, R. (2000). Enterprise resource planning: The role of the CIO and IT function in ERP. Communications of the ACM, 43 (4), 32-38.

Winters, D. B., Lindley, J. T., Topping, S., \& Lindley, L. T. (2008). The hidden financial costs of ERP software. Managerial Finance, 34 (2), 78-90.

\section{Author Response}

The authors of the paper under review value the positive contributions made and thank their colleague for his contribution.

Copyright: (c) $2016 \mathrm{Lim}$. This is an open-access article distributed under the terms of the Creative Commons Attribution-NonCommercial 3.0 Australia License, which permits noncommercial use, distribution, and reproduction in any medium, provided the original author and AJ IS are credited. 


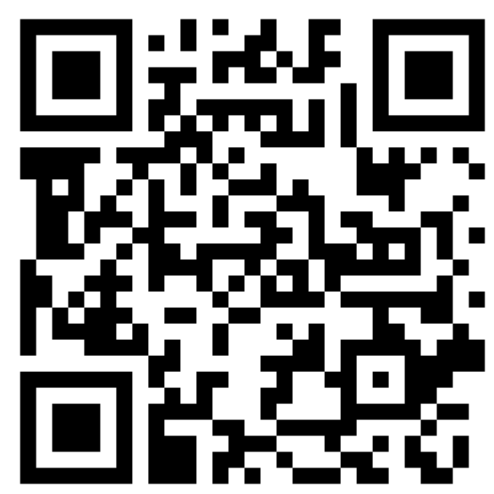

